PROCEEDINGS OF THE

AMERICAN MATHEMATICAL SOCIETY

Volume 125, Number 1, January 1997, Pages 287-292

S 0002-9939(97)03641-1

\title{
SMOOTH STRUCTURES ON COMPLEX SURFACES WITH FUNDAMENTAL GROUP $\mathrm{Z}_{2}$
}

\author{
SHUGUANG WANG \\ (Communicated by Ronald Stern)
}

\begin{abstract}
It is shown that the quotients of a complex surface under free holomorphic and anti-holomorphic involutions are homeomorphic but not diffeomorphic. This gives a way to construct exotic smooth structures on some complex surfaces.
\end{abstract}

\section{IntRoduction AND THE MAIN RESUlt}

For a complex surface $X$, it is possible to find an "exotic" smooth structure on the underlying topological manifold. In other words there may be a smooth oriented 4-manifold $X^{\prime}$ which is homeomorphic but not diffeomorphic to $X$. Each such pair of manifolds would in particular disprove the $h$-cobordism conjecture, and the first example, proved in Donaldson's fundamental paper [3], is provided by the Dolgachev surface $D_{2,3}$ and $\mathbf{C P}^{2} \# 9 \overline{\mathbf{C P}}^{2}$. The search for smooth structures on other simply-connected Dolgachev surfaces as well as on other simply-connected complex surfaces has been one of the focal points of studies in gauge theory.

In the case of non-simply-connected complex surfaces, results are also obtained by a number of people, notably in the serial papers by I. Hambleton and M. Kreck [10]-[12]. Nevertheless this case has so far received less attention than the simplyconnected case.

For non-simply-connected Dolgachev surfaces, the differentiable classification is given by Lübke-Okonek [16] and Maier [17], extending the simply-connected case of Friedman-Morgan [9] and Okonek-Van de Ven [20]. By using their topological classification for these surfaces, Hambleton and Kreck [10] establish that any Dolgachev surface has infinitely many smooth structures given by other Dolgachev surfaces. At the same time, Okonek [19] finds infinitely many smooth structures on the Enriques surface using homotopy K3 surfaces. Next Hambleton and Kreck [11] show that in general any complex surface with finite fundamental group has an exotic smooth structure provided its Euler characteristic is large enough, and this condition is finally removed in [12] when the surface is an elliptic surface or has a cyclic fundamental group.

Received by the editors July 1, 1995.

1991 Mathematics Subject Classification. Primary 57R55, 57R57, 57N13.

Work supported by the Research Board grant of the University of Missouri.

(C)1997 American Mathematical Society 
It is perhaps worth observing that the main point of the work of HambletonKreck [10]-[12] appears to be the classification of topological 4-manifolds with finite fundamental group, which is a delicate problem and still not completely resolved (a remaining case is the finite fundamental group of even order). Once the topological classification is obtained, the existence of exotic smooth structures on complex surfaces follows from some more or less direct applications of Donaldson's vanishing theorem [4]. As a result, the exotic smooth structures in [11]-[12] mostly come from manifolds of connected sums $Y_{1} \# Y_{2}$ where both $b_{2}^{+}\left(Y_{i}\right)>0$. (Here $b_{2}^{+}\left(Y_{i}\right)$ stands for the number of positive eigenvalues of the intersection form on $H_{2}\left(Y_{i}, \mathbf{Z}\right)$.)

In this paper we give another method to construct exotic smooth structures on some complex surfaces with fundamental group $\mathbf{Z}_{2}$. Even though the proof has the same flavor as $[11,12]$, here we invoke a different vanishing theorem from Wang [21], which does not directly involve the connected-sum manifolds like the above. Indeed, as will be remarked below, the new exotic smooth structures do not come from such connected sums.

Recall that a smooth map $\sigma$ between two almost complex manifolds is called anti-holomorphic if $\sigma_{*} J_{1}=-J_{2} \sigma_{*}$ on the tangent bundles, where $J_{1}, J_{2}$ are the almost complex structures of the manifolds (underlying some Kähler manifolds for the following discussions). In what follows $K$ denotes the canonical bundle of a Kähler manifold.

Theorem. Let $\widetilde{X}$ be a simply-connected Kähler surface, and suppose that $\tau, \sigma$ are two free involutions on $\widetilde{X}$, which are respectively holomorphic and anti-holomorphic.

(1) If $K_{\widetilde{X}}^{2}>0$ and $b_{2}^{+}(\widetilde{X})>3$, then the quotient manifolds $X=\widetilde{X} / \tau, X^{\prime}=\widetilde{X} / \sigma$ are not diffeomorphic to each other.

(2) If $X$ is not spin, then $X$ and $X^{\prime}$ are homeomorphic to each other.

Proof. (1) By averaging there is a metric and compatible Kähler form preserved by $\tau$; thus the quotient manifold $X$ has an induced Kähler structure. Moreover for the double cover $\widetilde{X} \rightarrow X$, the familiar formulae $e_{\widetilde{X}}=2 e_{X}, s_{\widetilde{X}}=2 s_{X}$ (Euler characteristic and signature) yield that $b_{2}^{+}(X)=\frac{1}{2}\left[b_{2}^{+}(\widetilde{X})-1\right]>1$. Hence by Witten [22], the Seiberg-Witten invariants on $X$ are defined and take a non-trivial value for the $\operatorname{spin}^{c}$ structure associated to the Kähler structure on $X$. On the other hand, it is shown in Wang [21] that the Seiberg-Witten invariants vanish for all $\operatorname{spin}^{c}$ structures on $X^{\prime}$. By the smooth invariance of the Seiberg-Witten invariants, $X$ and $X^{\prime}$ are consequently not diffeomorphic.

(2) Note that $\pi_{1}(X)=\pi_{1}\left(X^{\prime}\right)=\mathbf{Z}_{2}$. Moreover $X$ and $X^{\prime}$ have the same signature and Euler characteristic using the above formulae as well as the same (even) type, implying that the intersection forms on $H_{2}(X, \mathbf{Z})$ and $H_{2}\left(X^{\prime}, \mathbf{Z}\right)$ modulo torsions are isomorphic. Thus, according to Hambleton-Kreck [12, Theorem C], it is enough to check that $X, X^{\prime}$ have the same $w_{2}$-type in order to show them to be homeomorphic.

Since $X, X^{\prime}$ have the same universal cover $\widetilde{X}$, having the same $w_{2}$-type simply means that $X, X^{\prime}$ have the same spin type. Therefore the proof is completed if we show that $X^{\prime}$ does not have a spin structure. This is established in the following general Lemma.

Lemma. Suppose that $\tilde{X}$ is a Kähler manifold and $\sigma$ is a free anti-holomorphic involution on $\widetilde{X}$. Then the quotient manifold $X^{\prime}=\widetilde{X} / \sigma$ admits no spin structure. 
Proof. Suppose otherwise that $X^{\prime}$ has a spin structure, which can then be pulled back to a $\sigma$-equivariant spin structure on $\widetilde{X}$. As spin structures on $\widetilde{X}$ are in one-one canonical correspondence with the (holomorphic) square roots of the line bundle $K_{\widetilde{X}}$ by Hitchin [14], this means that $\sigma$ can be lifted to an involution on one of the square roots and consequently can be lifted to a bundle isomorphism on $K_{\widetilde{X}}$. In particular $\sigma^{*} c_{1}\left(K_{\widetilde{X}}\right)=c_{1}\left(K_{\widetilde{X}}\right)$ for the Chern class of $K_{\widetilde{X}}$. This is however seen to be a contradiction as follows. Being an anti-holomorphic involution, $\sigma$ induces a type-reversing map on the space of $(p, q)$-forms on $\widetilde{X}$, that is, $\sigma^{*}(\omega)$ is a $(q, p)$-form for any $(p, q)$-form $\omega$ on $\tilde{X}$. Therefore applying to $(0, n)$-forms $\left(\operatorname{dim}_{\mathbf{C}} \tilde{X}=n\right)$, we have $\sigma^{*} c_{1}\left(K_{\widetilde{X}}\right)=-c_{1}\left(K_{\widetilde{X}}\right)$, which contradicts the early formula.

On a complex curve, any anti-holomorphic involution is orientation-reversing. When the involution is free, the quotient is therefore a closed non-orientable surface on which no spin structure exists by definition. As a more instructive example for the Lemma, there is a free anti-holomorphic involution on $\mathbf{C P}^{1} \times \mathbf{C P}^{1}$ which is smoothly equivalent to the antipodal involution on $S^{2} \times S^{2}$; the quotient manifold is indeed not spin according to Habegger [13].

Remarks. (1) In the second part of the Theorem, the condition that $X$ be non-spin is satisfied if either $\widetilde{X}$ is non-spin or the signature of $\widetilde{X}$ is not divisible by 32 . In this sense, the Theorem can be applied to "most" simply-connected Kähler surfaces. Furthermore the simple-connectedness may be relaxed so that $\pi_{1}(X)$ and $\pi_{1}\left(X^{\prime}\right)$ are cyclic as [12, Theorem C] continues to hold for such groups.

(2) Since Seiberg-Witten invariants are preserved in blow-ups at points, namely connected sums with $\overline{\mathbf{C P}}^{2}$ (see Fintushel-Stern [8]), the quotient manifolds in the Theorem remain non-diffeomorphic after blowing-up. Compare with the Conjecture in Hambleton-Kreck [11].

(3) One interpretation of the Theorem is that the quotient complex surface $X$ admits an exotic smooth structure from $X^{\prime}$. Note that $X^{\prime}$ can not smoothly be decomposed as a connected sum $Y_{1} \# Y_{2}$ with $b_{2}^{+}\left(Y_{i}\right)>0$ by Wang [21]. (Furthermore, $X^{\prime}$ is irreducible according to Kotschick [15].) Thus this kind of smooth structure on $X$ has a new feature compared with Hambleton-Kreck [11, 12], where the smooth structures are from connected-sum manifolds.

(4) Another interpretation, of especially the first part of the Theorem, is that holomorphic and anti-holomorphic free involutions are always differentiably nonequivalent. Such exotic free involutions on smooth manifolds are already obtained before the appearance of gauge theory, in Cappell-Shaneson [2] and Fintushel-Stern [7]. So the Theorem here may also be viewed as an extension of these works. By way of mentioning, the condition $b_{2}^{+}(\widetilde{X})>3$ in the first part of the Theorem can not be dropped, as Donaldson [5] observes that any anti-holomorphic involution on a K3 surface (with $b_{2}^{+}=3$ ) is equivalent smoothly to a holomorphic involution.

(5) From the argument of the Theorem and the Lemma, one sees that any two free anti-holomorphic involutions on the same simply-connected Kähler surface always give rise to homeomorphic quotient manifolds. It is interesting to examine when the quotients are also diffeomorphic to each other. Compare with Finashin-Kreck-Viro [6], where they find non-diffeomorphic knottings from different anti-holomorphic involutions on (different) Dolgachev surfaces although the quotient manifolds are all diffeomorphic to $S^{4}$. 


\section{EXAMPLES}

It is a strong restriction for complex surfaces to admit both holomorphic and anti-holomorphic involutions. In view of this, it is of interest to give examples satisfying the conditions in the Theorem above.

Example. Here we will give a family of simply-connected complex surfaces as branched double covers of $Y=\mathbf{C P}^{1} \times \mathbf{C P}^{1}$, each of which has free holomorphic and anti-holomorphic involutions.

For any pair of integers $k, l$, consider a complex curve $A=f^{-1}(0)$ on $Y$ given by a bi-homogeneous polynomial of even degree

$$
f\left(x_{0}, x_{1} ; y_{0}, y_{1}\right)=x_{0}^{2 k} y_{0}^{2 l}+x_{0}^{2 k} y_{1}^{2 l}+x_{1}^{2 k} y_{0}^{2 l}+x_{1}^{2 k} y_{1}^{2 l} .
$$

Since $0=[A] \in H_{2}\left(Y, \mathbf{Z}_{2}\right)$, there is a (unique) double cover $p: \widetilde{X} \rightarrow Y$ branched along $A$. From the standard formula in Barth-Peters-Van de Ven [1, page 182] relating the canonical bundles on any double cover, one has $K_{\widetilde{X}}^{2}=4(k-2)(l-2)$ which is positive once $k, l>2$.

In order to define involutions on $\tilde{X}$, it is most convenient to use an explicit construction of $\widetilde{X}$ from, for instance, Matsuoka [18]. Let $x=x_{0}: x_{1}: x_{2} \in \mathbf{C P}^{2}$ be a typical point and $U=\left\{x \in \mathbf{C P}^{2} \mid\right.$ either $x_{0}$ or $\left.x_{1} \neq 0\right\}$. Set $V=U \times U \subset$ $\mathbf{C P}^{2} \times \mathbf{C P}^{2}$, then one has the description

$$
\widetilde{X}=\left\{(x, y) \in V \mid f\left(x_{0}, x_{1} ; y_{0}, y_{1}\right)-x_{2}^{2 k} y_{2}^{2 l}=0\right\} / \sim,
$$

where the equivalence relation $\sim$ is defined by

$$
\left(x_{0}: x_{1}: x_{2}, y_{0}: y_{1}: y_{2}\right) \sim\left(x_{0}: x_{1}:\left(r x_{2}\right), y_{0}: y_{1}:\left(s y_{2}\right)\right)
$$

for any $r, s \in \mathbf{C}$ such that $r^{k} s^{l}=1$. Let $[x, y] \in \widetilde{X}$ denote the equivalence class of $(x, y) \in V$; the covering map $p: \widetilde{X} \rightarrow Y$ is then just the projection $[x, y] \mapsto$ $\left(x_{0}: x_{1}, y_{0}: y_{1}\right)$. With this concrete construction, $\widetilde{X}$ has a natural complex structure, and it is moreover simply connected by an elementary topological argument, using that the elements of $\pi_{1}\left(\widetilde{X} \backslash p^{-1}(A)\right)$ can be represented by loops surrounding $p^{-1}(A)$ in $\widetilde{X}$.

Take any $a, b \in \mathbf{C}$ satisfying $a^{k} b^{l}=-1$. A specific choice of $a, b$ will not affect the maps introduced below because of the equivalence relation $\sim$. First the deck transformation $\theta$ of the cover $\widetilde{X} \rightarrow Y$ has the form:

$$
\theta([x, y])=\left[x_{0}: x_{1}:\left(a x_{2}\right), y_{0}: y_{1}:\left(b y_{2}\right)\right] .
$$

As a lifting of the complex conjugation on $Y$, one can take

$$
\sigma([x, y])=\left[\bar{x}_{0}: \bar{x}_{1}: a \bar{x}_{2}, \bar{y}_{0}: \bar{y}_{1}: b \bar{y}_{2}\right],
$$

which is easily seen to be a free anti-holomorphic involution on $\widetilde{X}$.

Suppose in addition that $k$ and $l$ are even. We can also put a free holomorphic involution $\tau$ on $\widetilde{X}$ defined by

$$
\tau([x, y])=\left[x_{0}:\left(-x_{1}\right):\left(a x_{2}\right), y_{0}:\left(-y_{1}\right):\left(b y_{2}\right)\right] ;
$$

it can be checked with a little patience that $\tau$ is free. (It is necessary to assume $k, l$ to be even in view of $b_{2}^{+}(\widetilde{X} / \tau)=\frac{1}{2}\left[b_{2}^{+}(\widetilde{X})-1\right] \equiv 1 \bmod 2$.) With the introduction of $\tau, \sigma$ on $\widetilde{X}$, we complete our Example. 
The point of the above Example is that both $\tau$ and $\sigma$ are liftings of some involutions on $Y$. Although these involutions on $Y$ have fixed points, their liftings on $\widetilde{X}$ can be free since their fixed point sets miss the branched locus $A$. More precisely, we can make use of the following observation:

Proposition. Let $Y$ be a smooth 4-manifold and $p: \widetilde{X} \rightarrow Y$ a double cover branched along a surface A. Suppose that $\rho: Y \rightarrow Y$ is a diffeomorphism satisfying:

(1) $\rho$ restricts to $A$,

(2) the fixed point set Fix $\rho$ of $\rho$ is connected and disjoint from $A$.

Then $\rho$ can be lifted to a diffeomorphism on $\widetilde{X}$ which does not have any fixed point.

Proof. Since $\rho$ preserves $A$, it can always be lifted to a diffeomorphism, say labeled as $r_{1}$, on $\tilde{X}$. Then $r_{2}=\theta r_{1}=r_{1} \theta$ is another lifting of $\rho$, where $\theta$ is the deck transformation of the branched cover $p: \widetilde{X} \rightarrow Y$. We want to show that one of the $r_{i}$ has no fixed point.

Suppose otherwise that $r_{i}$ has a fixed point $x_{i}$ for $i=1,2$. Then $y_{i}=p\left(x_{i}\right)$ are the fixed points of $\rho$ and hence, by assumption, they can be connected by a path $\gamma$, which lies entirely in Fix $\rho$ and does not intersect $A$. Lift $\gamma$ to a path $\Gamma$ in $\widetilde{X} \backslash p^{-1}(A)$ starting at $x_{1}$ and ending at $x_{2}^{\prime}$. Then it is easy to check $r_{1}\left(x_{2}^{\prime}\right)=\theta\left(x_{2}^{\prime}\right)$ from $p\left(x_{2}^{\prime}\right)=p\left(x_{2}\right)$ and $r_{1}\left(\theta\left(x_{2}\right)\right)=r_{2}\left(x_{2}\right)=x_{2}$. Since $\gamma \subset \operatorname{Fix} \rho$ and is away from $A$, one sees that the set of points on $\Gamma$ has a disjoint decomposition of non-empty closed subsets:

$$
\Gamma=\left\{x \in \Gamma \mid r_{1}(x)=x\right\} \cup\left\{x \in \Gamma \mid r_{1}(x)=\theta(x)\right\} .
$$

This of course contradicts that $\Gamma$ is connected.

Thus, if $\rho$ in the Proposition is a holomorphic or anti-holomorphic involution on a complex surface $Y$ and can be lifted to an involution on $\widetilde{X}$ (in general liftings may be order four), then there is a free holomorphic or anti-holomorphic lifting involution. So by using suitable branched covers, one can obtain many examples of complex surfaces admitting both free holomorphic and anti-holomorphic involutions.

Note that in the previous Example as branched cover of $Y=\mathbf{C P}^{1} \times \mathbf{C P}^{1}$, the holomorphic map $(x, y) \mapsto\left(x_{0}:\left(-x_{1}\right), y_{0}:\left(-y_{1}\right)\right)$ on $Y$ has four fixed points and can still be lifted to the free involution $\tau$. This shows that the assumption that Fix $\rho$ is connected is by no means necessary for the Proposition, but the author is unable to remove this assumption for a general case.

Finally there is a specific case to consider concerning the question in Remark (5) of Section 1:

Question. Suppose that $k+l$ is even in the Example above. On the surface $\widetilde{X}$, do the two free anti-holomorphic involutions

$$
\begin{gathered}
{[x, y] \mapsto\left[\bar{x}_{0}: \bar{x}_{1}: a \bar{x}_{2}, \bar{y}_{0}: \bar{y}_{1}: b \bar{y}_{2}\right],} \\
{[x, y] \mapsto\left[\bar{x}_{1}:\left(-\bar{x}_{0}\right): \bar{x}_{2}, \bar{y}_{1}:\left(-\bar{y}_{0}\right): \bar{y}_{2}\right]}
\end{gathered}
$$

have diffeomorphic quotients? (Note that the two involutions push down to nonequivalent involutions on $Y$ as one has fixed points $\mathbf{R} \mathbf{P}^{1} \times \mathbf{R} \mathbf{P}^{1}$ and the other is free.) 


\section{REFERENCES}

1. W. Barth, C. Peters and A. Van de Ven, Compact complex surfaces, Springer-Verlag, 1984. MR 86c: 32026

2. S. Cappell and J. Shaneson, Some new four-manifolds, Ann. Math. 104 (1976), 61-72. MR 54:6167

3. S. Donaldson, Irrationality and the h-cobordism conjecture, J. Diff. Geom. 26 (1987), 141-168. MR 88j:57035

4. _ Polynomial invariants for smooth four-manifolds, Topology 29 (1990), 257-315. MR 92a: 57035

5. - Yang-Mills invariants of four-manifolds, Geometry of low-dimensional manifolds, Vol. 1, pp. 5-41. S. Donaldson and C. Thomas eds., Cambridge University Press, 1990. MR 93f: 57040

6. S. Finashin, M. Kreck and O. Viro, Non-diffeomorphic but homeomorphic knottings in the 4-sphere, Rohklin Seminar, O. Viro, ed., Lecture Notes in Math., Vol. 1346, Springer-Verlag, 1988. MR 90h:57021

7. R. Fintushel and R. Stern, An exotic free involution on $S^{4}$, Ann. Math. 113 (1981), 357-365. MR 84g:57036

8. _ Rational blowdowns of smooth 4-manifolds, Preprint.

9. R. Friedman and J. Morgan, On the diffeomorphism type of certain algebraic surfaces I, J. Diff. Geom. 27 (1988), 297-369. MR 89d:57046

10. I. Hambleton and M. Kreck, Smooth structures on algebraic surfaces with cyclic fundamental group, Invt. Math. 91 (1988), 53-59. MR 89a:57043

11. 102 (1990), 109-114. MR 91g:57032

12. _ Cancellation, elliptic surfaces and the topology of certain four-manifolds, J. Reine Angew. Math. 444 (1993), 79-100. MR 95h:57036

13. N. Habegger, Une variete de dimension 4 arec forme d'intersection paire, Comp. Math. 63 (1987), 217-222.

14. N. Hitchin, Harmonic spinors, Adv. Math. 14 (1974), 1-55. MR 50:11332

15. D. Kotschick, On irreducible four-manifolds, to appear.

16. M. Lübke and C. Okonek, Differentiable structures on elliptic surfaces with cyclic fundamental group, Comp. Math. 63 (1987), 217-222. MR 89d:57048

17. F. Maier, On the diffeomorphism type of elliptic surfaces with cyclic fundamental group, Tulane University Thesis (1987).

18. S. Matsuoka, Non-singular algebraic curves in $\mathbf{R P}^{1} \times \mathbf{R} \mathbf{P}^{1}$, Trans. Amer. Math. Soc. 324 (1991), 87-107. MR 91b:14054

19. C. Okonek, Fake Enriques surfaces, Topology 27 (1988), 415-427. MR 90e:57062

20. C. Okenek and A. Van de Ven, Stable vector bundles and differential structures on certain elliptic surfaces, Invt. Math. 86 (1988), 357-370.

21. S. Wang, A vanishing theorem for Seiberg-Witten invariants, Math. Res. Letters 2 (1995), 305-311. MR 96c:57056

22. E. Witten, Monopoles and four-manifolds, Math. Res. Letters 1 (1994), 769-796. MR 96d: 57035

Department of Mathematics, University of Missouri, Columbia, Missouri 65211

E-mail address: sw@wang.cs.missouri.edu 